\title{
Separation of the proximal humeral epiphysis
}

\author{
Alaric Aroojis, Harold D’Souza, M G Yagnik
}

A 42-month-old girl presented with a history of sudden onset swelling of the left shoulder of 4-5 days duration with no preceding history of significant trauma or fall. The child was irritable and mildly febrile on examination. A tense globular swelling measuring $6 \times 5 \times 5 \mathrm{~cm}$ was present in the left shoulder region which was tender and fluctuant on palpation (figure 1). There was an increase in the local temperature and the skin overlying the swelling was stretched and glossy. Shoulder movements were painful and restricted. There was no regional lymphadenopathy nor any distal neurovascular compromise. Other joints were normal and no abnormality was detected in other systems. The child had chronic non-pitting oedema of both legs and was reluctant to walk. There was no history of a bleeding disorder nor was any such family history elicitable. An $\mathrm{X}$-ray of the left shoulder revealed a complete separation of the proximal humeral epiphysis with osteopenia and surrounding soft tissue swelling (figure 2).

Haemogram revealed anaemia (haemoglobin $5.3 \mathrm{~g} / \mathrm{dl}$ ) but a normal leucocyte count of $7.2 \times$ $10 \%$ (polymorphs $44 \%$, lymphocytes $48 \%$, and eosinophils $8 \%$ ). Reticulocyte count was $1 \%$, platelet count was adequate, and the erythrocyte sedimentation rate was $10 \mathrm{~mm}$ at one hour. Bleeding time, clotting time and other tests for bleeding profile were within normal limits. Serum calcium and phosphorus levels were normal but alkaline phosphatase level was elevated to 306 IU/1 (normal range 80-180 IU/1).

Needle biopsy of the swelling resulted in the evacuation of approximately $20 \mathrm{ml}$ of altered blood and a few soft tissue and bony fragments which on microscopic examination revealed the presence of proliferating fibroblasts, spicules of necrotic bone and abundant vascular proliferation. A single X-ray of the knee (figure 3), however, showed typical changes which aided in the diagnosis of this condition.

\author{
Department of \\ Paediatric \\ Orthopaedics, Bai \\ Jerbai Wadia Hospital \\ for Children, Parel, \\ Mumbai - 400012, \\ India \\ A Aroojis \\ H D'Souza \\ M G Yagnik \\ Correspondence to \\ Dr Alaric Aroojis, 8/424 \\ Church View, 14th Road \\ TPS 3, Bandra, Mumbai - \\ 400050, India
}

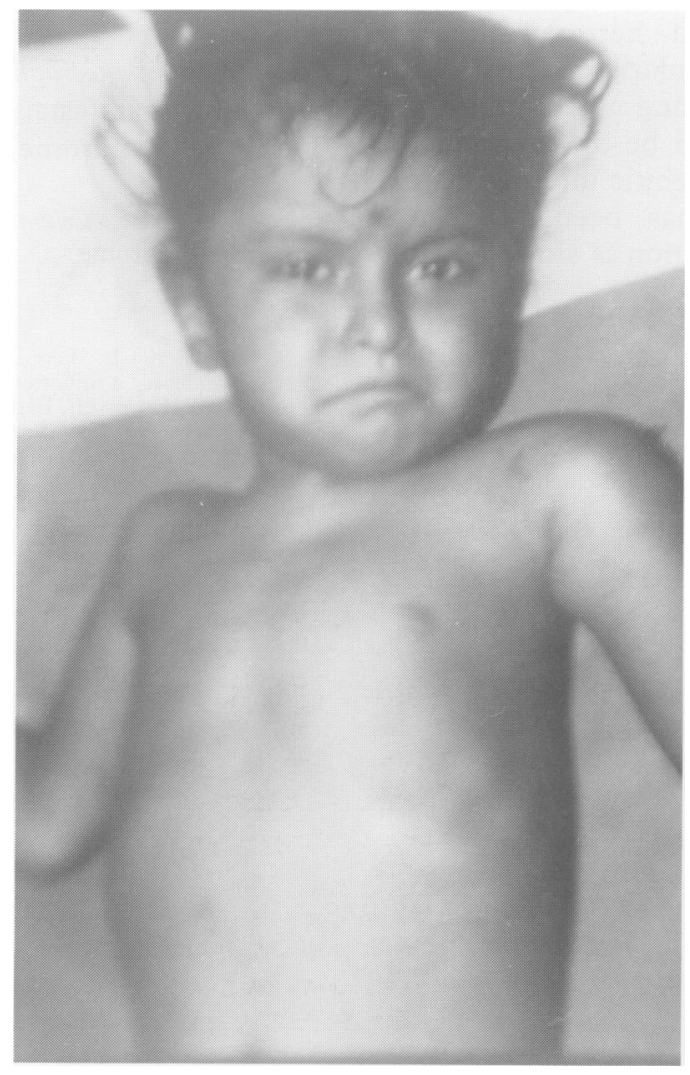

Figure 1 Clinical photograph

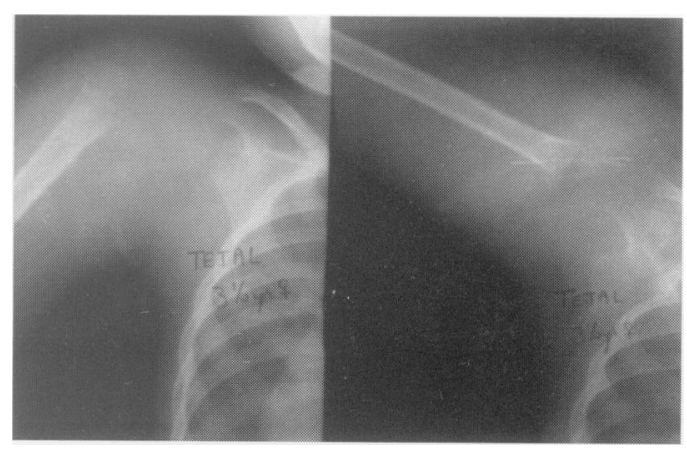

Figure 2 X-Ray of left shoulder 


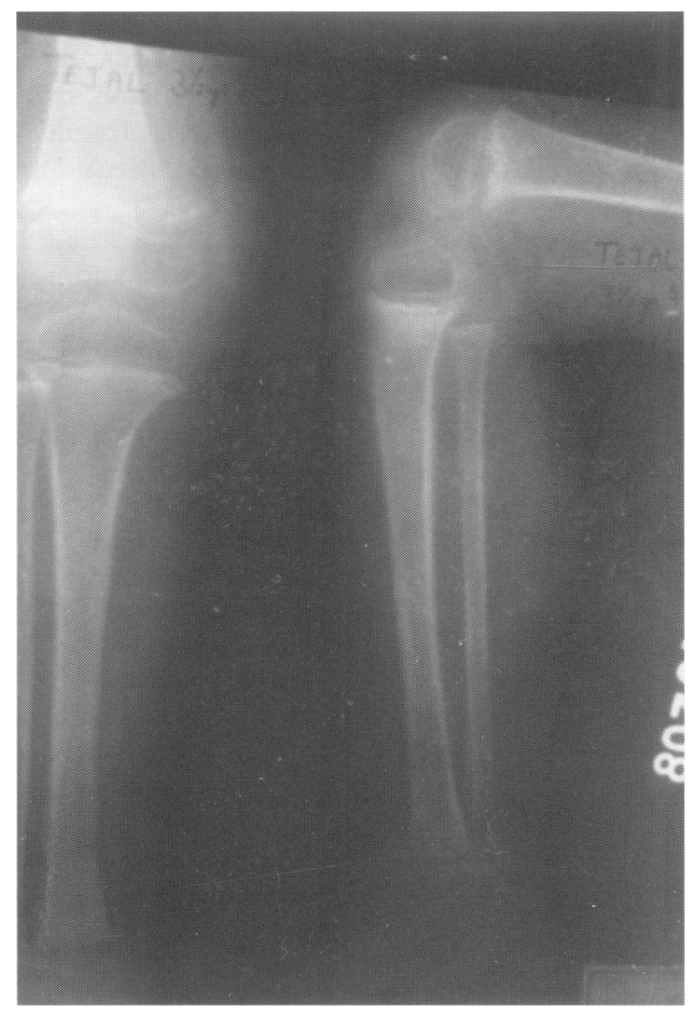

Figure 3 X-Ray of knee and leg

\section{Questions}

1 What is the most probable diagnosis?

2 What characteristic findings are seen in figure 3?

3 What is the underlying pathological process ?

4 What is the management of the proximal humeral epiphyseal separation in this condition ? 


\section{Answers}

\section{QUESTION 1}

The clinical and radiographic features are consistent with the diagnosis of scurvy or vitamin C deficiency, causing spontaneous epiphyseal separation and subperiosteal haemorrhage. Other differential diagnosis to be considered in view of the pain, local tenderness and pseudoparalytic state are listed in the box.

\begin{tabular}{|l|}
\hline Scurvy: differential diagnosis \\
\hline - acute osteomyelitis \\
- septic arthritis \\
- bleeding disorders \\
- Henoch-Schonlein purpura \\
- thrombocytopenic purpura \\
- leukaemia \\
- acuilis rheumatic fever \\
\hline
\end{tabular}

QUESTION 2

Radiographic changes typical of scurvy ${ }^{12}$ are best visualised at the knees, wrists and costochondral junctions, all sites of rapid bone growth. The initial picture is that of generalised osteoporosis of the spongiosa and a decrease in the cortical thickness. There is an increase in the width and opacity of the zone of provisional calcification at the ends of the metaphysis (Fraenkel's white line) and around the margins of the epiphyseal centres of ossification (Wimberger's ringing of epiphysis). Outward projection of the zone of provisional calcification beyond the limits of the shaft, produces lateral spur formation at the ends of the metaphysis (Pelkan's spur). ${ }^{3}$ The 'scurvy line' or scorbutic zone is seen as a transverse band of decreased density adjacent to the dense zone of provisional calcification. Subperiosteal haemorrhage is another distinctive sign; the lower ends of the femur and tibia and the upper ends of the humerus being frequent sites. Spontaneous epiphyseal separations may occasionally occur. $^{45}$

QUESTION 3

Skeletal changes in scurvy have been studied in the bones of both animals and humans. ${ }^{67}$ Ascorbic acid deficiency causes a dysfunction of the osteoblasts; this has been ascribed to a loss of ribonucleic acid and the disappearance of cytochrome oxidase activity in the cytoplasm of the osteoblasts. ${ }^{7}$ The result is a failure to produce osteoid tissue and form new bone. The chondroblasts, however, continue to proliferate normally, align in columns and form chondroid tissue. There is no disturbance in mineralisation; the degenerating cartilage columns calcify normally but are not converted into bone. ${ }^{3}$ The plump osteoblasts become flattened and resemble connective tissue fibroblasts. ${ }^{6}$
The bone trabeculae and cortices of long bones are thin and the zone of provisional calcification, through which epiphyseal separation may occur, is fragile. Collagen synthesis is also interfered with and instead of normal collagenised fibrous tissue, a primitive type of connective tissue is formed. In experimental studies in guinea pigs, a suppression of the entire growth zone, eventually leading to complete cessation of endochondral ossification, has been demonstrated. ${ }^{6}$

\section{QUESTION 4}

Rapid recovery occurs following daily oral or parenteral administration of ascorbic acid (vitamin C) at a dose of 100-200 mg per day. Pain and tenderness disappear, subperiosteal haemorrhages calcify and body growth soon resumes.

Separation of the epiphysis in scurvy is best treated conservatively by splintage and observation and a closed or open reduction of the displaced epiphysis is rarely required. Complete recovery by remodelling is the rule and residual deformity or disturbance in longitudinal bone growth are seldom seen. ${ }^{8}$

\section{Discussion}

The clinical manifestations of scurvy have been well chronicled in the literature since 1757, when James Lind published $A$ Treatise on the Scurvy and recommended the inclusion of lemon juice in naval diets. Scurvy develops following 6-12 months of dietary vitamin C deficiency. Early manifestations consist of loss of appetite, irritability and a slow gain in weight. With the progression of scurvy, the typical scorbutic signs appear. Haemorrhage of the gums, especially around the upper incisors is common. Subperiosteal haemorrhage is yet another distinctive sign, the lower ends of the femur, tibia and humerus being frequent sites. The child is very apprehensive and prefers to lie supine in a frog-leg position - a pseudoparalytic posture in response to pain. The legs are swollen and oedematous. Spontaneous epiphyseal separation may take place. Beading of the ribs at costochondral junctions may develop. Haemorrhages may occur in subcutaneous tissue, in and around muscles in the urinary or gastrointestinal tract or in the subchondral space. Anaemia and delayed wound healing are common. ${ }^{3}$

Fractures of the calcified cartilage of the epiphyseal plate and epiphyseal separations are known to occur in scurvy, but have rarely been reported in literature. ${ }^{48}$ The management of epiphyseal separation in scurvy is controversial, although there appears to be a general consensus that permanent or residual deformity or disturbance of longitudinal bone growth is rare. ${ }^{68}$ Notwithstanding the often extensive skeletal involvement, the lesions heal promptly following adequate dietary treatment. This can be explained by the fact that the periosteum has its strongest attachment at the growth plate, which is detached together with the epiphysis. The epiphysis therefore, remains lined up to the denuded shaft by a periosteal 
envelope containing blood (subperiosteal haemorrhage). Upon administration of ascorbic acid, the subperiosteal haemorrhage rapidly calcifies and remodels within a few months leaving no residual deformity or growth disturbance. Furthermore, epiphyseal separation in scurvy occurs through the relatively avascular zone of provisional calcification causing little or no damage to the epiphyseal or metaphyseal blood vessels supplying the growth plate. ${ }^{8}$

A one-year follow-up of our case (figure 4) amply demonstrates the intense remodelling potential following vitamin $\mathrm{C}$ supplementation and dietary advice, and confirms the fact that an open or closed reduction of the displaced epiphysis is rarely required.

\section{Final diagnosis}

Scurvy causing proximal humeral epiphyseal separation.

1 Brailsford JW. Some radiological manifestations of early scurvy. Arch Dis Child 1953;28:31.

Bromer RS. A critical analysis of the roentgen signs of infantile scurvy. $A \Im R$ 1943;49:575.

3 Tachdjian MO Pediatric orthopedics, vol 2, 2nd edn. Philadelphia: WB Saunders, 1990; p 918.

4 Nerubay J, Pilderwasser D. Spontaneous bilateral distal femoral physiolysis due to scurvy. Acta Orthopaed Scand 1984;55:18.

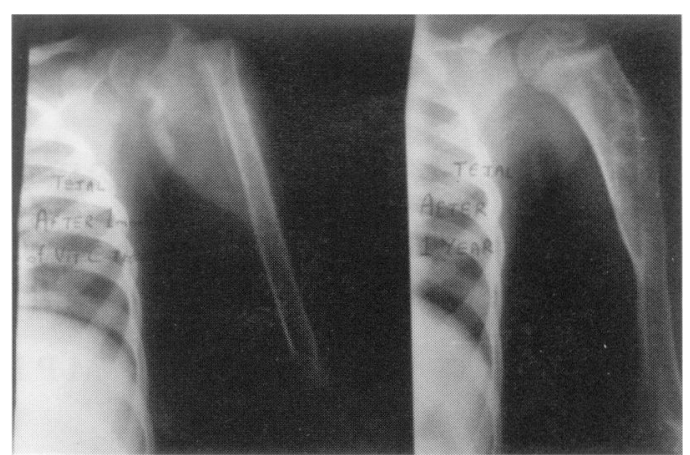

Figure 4 Complete remodelling of the proximal humerus following vitamin $\mathrm{C}$ therapy for 12 months

Keywords: scurvy; epiphyseal separation; proximal humerus

We thank the Dean, Bai Jerbai Wadia Hospital for Children, for permission to utilise hospital data.

5 Silverman FN. An unusual osseous sequel to infantile scurvy. F Bone foint Surg 1953;35-A:215.

6 Banks SW. Bone changes in acute and chronic scurvy. An experimental study. $\mathcal{F}$ Bone foint Surg 1943;25:553.

Follis RH. Histochemical studies on cartilage and bone. II. Ascorbic acid deficiency. Bull fohn Hopkins Hosp 1951;89:9. 8 Silverman FN. Recovery from epiphyseal invagination. Sequel to an unusual complication of scurvy. $\mathcal{f}$ Bone foint Surg 1970;52-A:384. 